\title{
Spanish translation, cross-cultural adaptation, and validation of the Questionnaire for Diabetes-Related Foot Disease (Q-DFD)
}

This article was published in the following Dove Press journal:

Vascular Health and Risk Management

27 August 2013

Number of times this article has been viewed

\section{Wilson Castillo-Tandazo \\ Adolfo Flores-Fortty \\ Lourdes Feraud \\ Daniel Tettamanti}

School of Medicine, Universidad Espíritu Santo - Ecuador, Samborondón, Guayas, Ecuador
Correspondence: Wilson Castillo-

Tandazo

Universidad Espíritu Santo -

Ecuador, School of Medicine,

Cdla. El Tornero III. Km.3 Vía

Samborondón, EC090I-952, Ecuador

$\mathrm{Tel}+59342835630$ ext 108

Fax +59342835483

Email wcastillo@uees.edu.ec
Purpose: To translate, cross-culturally adapt, and validate the Questionnaire for DiabetesRelated Foot Disease (Q-DFD), originally created and validated in Australia, for its use in Spanish-speaking patients with diabetes mellitus.

Patients and methods: The translation and cross-cultural adaptation were based on international guidelines. The Spanish version of the survey was applied to a community-based (sample A) and a hospital clinic-based sample (samples B and C). Samples A and B were used to determine criterion and construct validity comparing the survey findings with clinical evaluation and medical records, respectively; while sample $\mathrm{C}$ was used to determine intra- and inter-rater reliability.

Results: After completing the rigorous translation process, only four items were considered problematic and required a new translation. In total, 127 patients were included in the validation study: 76 to determine criterion and construct validity and 41 to establish intra- and inter-rater reliability. For an overall diagnosis of diabetes-related foot disease, a substantial level of agreement was obtained when we compared the Q-DFD with the clinical assessment (kappa 0.77, sensitivity $80.4 \%$, specificity $91.5 \%$, positive likelihood ratio [LR+] 9.46, negative likelihood ratio [LR-] 0.21); while an almost perfect level of agreement was obtained when it was compared with medical records (kappa 0.88 , sensitivity $87 \%$, specificity 97\%, LR+29.0, LR- 0.13). Survey reliability showed substantial levels of agreement, with kappa scores of 0.63 and 0.73 for intra- and inter-rater reliability, respectively.

Conclusion: The translated and cross-culturally adapted Q-DFD showed good psychometric properties (validity, reproducibility, and reliability) that allow its use in Spanish-speaking diabetic populations.

Keywords: diabetes mellitus, peripheral vascular disease, diabetic neuropathy, foot ulcers

\section{Introduction}

Diabetes mellitus (DM) remains the most common of the chronic metabolic diseases, with 285 million adults affected worldwide in 2010. This is estimated to increase to 439 million adults affected by 2030 , with prevalence rising from $6.4 \%$ to $7.7 \% .^{1}$ In Ecuador, the World Health Organization predicts a prevalence of 921,000 diabetic patients by $2030 .^{2}$ In addition, the National Institute of Statistics and Census of Ecuador determined in 2010 that DM was the second cause of mortality in the country, at $6.5 \%{ }^{3}$

Diabetes-related foot disease (DRFD) is one of the main complications of DM and consists of several pathologies, including peripheral vascular disease (PVD), diabetic neuropathy (DN), Charcot's neuroarthropathy, foot ulcerations, osteomyelitis, and limb amputation. ${ }^{4}$ Among diabetic patients, the prevalence of foot ulcers ranges from 
$4 \%$ to $10 \%$, with a lifetime risk as high as $25 \% .{ }^{5}$ Amputations due to DRFD are preceded by ulceration in $85 \%$ of cases, and carry a survival rate of around $50 \%$ after 3 years. ${ }^{6-8}$

In Ecuador, a previous study showed a prevalence of $11 \%$ for DRFD. ${ }^{9}$ Given its high morbi-mortality, early detection is crucial. Although clinical evaluation remains as the gold standard, it is cost- and time-consuming and not widely available in rural populations. To address these issues, many surveys concerning DRFD have been developed. However, most of them only evaluate DN or PVD as individual components of DRFD. After an extensive search, we found that the Questionnaire for Diabetes-Related Foot Disease (Q-DFD) is the only survey that addresses all the components of DRFD within one tool, therefore, we chose this survey for the present study. ${ }^{10}$

The Q-DFD was created in English and validated in an Australian population. The aim of this study was to translate, cross-culturally adapt, and validate the Q-DFD for its use in Spanish-speaking diabetic patients in Ecuador.

\section{Materials and methods}

This study, designed as a cross-sectional model, was approved by the Medical Ethics Committee of the Universidad Central del Ecuador. Written and verbal informed consent was obtained from all participants prior to their inclusion in this study. Funding for this project was granted by the Research Center of Universidad Espiritu Santo.

\section{The Q-DFD}

The first author of the Q-DFD was contacted and asked for permission and collaboration with the development of this project. This questionnaire was created in the year 2009 by Bergin et $\mathrm{al}^{10}$ and comprises 12 questions referring to signs and symptoms associated with PVD, DN, amputations, deformities, and foot ulcers.

\section{Translation}

The translation and cross-cultural adaptation were carried out based on the guidelines and international criteria proposed by Sperber. ${ }^{11}$

\section{Forward translation}

The Q-DFD was translated independently from English into Spanish by two professional translators. Each one provided a written report, which was combined by the authors in a new version that contained the most reliable translation for each question.

\section{Back translation}

The new version was back translated to English by two different professional translators, who were blinded to the original version of the Q-DFD. A new English version was developed by combining the written reports. The aim of the back translation process was to compare each item of the new English version with the original Q-DFD.

\section{Cross-language validation}

Each item of the new English version was compared with the original questionnaire by 30 raters fluent in English. The success of the translation was evaluated using two scales of comparison: comparability of language and similarity of interpretability. Each one used Likert scales ranging from 1 (extremely comparable/extremely similar) to 7 (not at all comparable/not at all similar). A mean score for each question was obtained. Questions with mean scores of three or less were included in the Spanish version. However, any questions with mean scores greater than three required a formal review and a retranslation until they indicated a valid version. The objective of this process was to identify potentially problematic items and retranslate them until the translated version was interpreted equally in both languages.

\section{Test of the pre-final version}

The Spanish version of the Q-DFD was tested on 16 subjects diagnosed with DM. Each subject provided feedback on the survey content, which was used to make final corrections. The authors discussed the information obtained and developed the final Spanish version.

\section{Setting and subjects}

A consecutive sample of 138 subjects was recruited and equally divided (46) into three samples: a community-based (sample A), and a hospital clinic-based sample (samples B and $C$ ). The inclusion criteria applied in all the samples were as follows: age $\geq 45$ years, a previous diagnosis of DM, permanent residents of Guayaquil, and enough speaking skills to complete a Spanish interview over the phone.

\section{Sample A}

This sample was recruited through advertisements in two local newspapers. The advertisements contained the inclusion criteria and a contact number for subjects interested in participating in the study. Those who contacted the investigators were asked for their names, telephone number, and date and time they preferred to be contacted. Prior to the survey being 
carried out, participants were asked for verbal consent. The survey was administered by a fourth-year medical student, and data were documented.

After the completion of the survey, subjects were invited to attend clinical evaluation. This was performed in an outpatient clinic by two specialized physicians (a neurologist and a vascular surgeon) with wide experience in these procedures. Data were recorded using a screening tool developed by Frykberg et al, ${ }^{12}$ and written informed consent was obtained prior to the clinical evaluation. The evaluation included assessment of DN using the pinprick test and determination of the Achilles reflex; assessment of PVD with manual palpation of pedal pulses and determination of Ankle Brachial Index (with an $8 \mathrm{mHz}$ hand-held Doppler, sphygmomanometer, and standard blood pressure cuff); and determination of foot deformity, foot ulcers, amputation, and ulceration.

\section{Sample B}

Sample B was recruited from attendees of an outpatient department at the Hospital Teodoro Maldonado Carbo located in Guayaquil. Members of the research staff assisted during consultation hours and invited patients who met inclusion criteria to participate in the study. For further analysis, sample B (B1) was divided into two groups: sample B2 (patients with known foot complications) and sample B3 (patients without known foot complications). After obtaining written informed consent, the presence of foot complications was determined by reviewing medical records looking for DN, PVD, amputation, and ulceration. Participants were asked to provide contact details to complete the Q-DFD over the telephone in the next few weeks. Verbal consent was confirmed prior to the survey being conducted.

\section{Sample C}

Sample C was used to evaluate the intra- and inter-rater reliability. The recruitment process in sample $\mathrm{C}$ was similar to that for sample B. Individuals who agreed to participate in the study completed the Q-DFD on three different occasions. Inter-rater reliability was obtained by comparing the first two interviews, which were conducted on the same day by two different interviewers. Both were blinded to each other's results. Intra-rater reliability was determined with the third interview, which was conducted by one of the first two interviewers 7 days later. Verbal consent was obtained prior to the survey being conducted.

\section{Quality control}

To guarantee that survey results were reliable, interviewers were asked to complete a training course. It was taught by the investigators and consisted of 10 hours regarding knowledge and familiarity with the survey, diction, interview conduction skills, and ability to interpret answers. Furthermore, all interviewers completed the Good Clinical Practice Course (https:// $\underline{\text { live.blueskybroadcast.com/bsb/client/CL DEFAULT.asp?C }}$ $\underline{\text { lient }=6 \& \mathrm{PCAT}=5169 \& \mathrm{CAT}=5169}$ ) prior to the beginning of the study.

\section{Statistical analyses}

The data collected were transferred from physical forms to electronic spreadsheets (Microsoft ${ }^{\circledR}$ Excel, version 2010; Microsoft Corporation, Redmond, WA, USA), and analyzed using SPSS software version 19.0 (Statistical Package for the Social Sciences; Chicago, IL, USA). Patient characteristics and descriptive data were calculated using means, frequencies, and standard deviations. Criterion and construct validity were estimated using kappa coefficient, sensitivity, specificity, and likelihood ratios (positive likelihood ratio [LR+] and negative likelihood ratio [LR-]). Prevalence rates for individual components of DRFD are reported as percentages. To interpret the kappa coefficient, the following definitions were used: 0 , poor agreement; 0.2 , slight agreement; $0.21-0.4$, fair agreement; $0.41-0.6$, moderate agreement; 0.61-0.8, substantial agreement; and $0.81-1$, almost perfect agreement. ${ }^{13}$

\section{Results \\ Translation}

Translation and back translation

During the translation and back translation process, minimal discrepancies were encountered.

\section{Cross-language validation}

Table 1 shows the items that needed a formal review of the translation after failing to obtain a mean score of at least three in relation to comparability of language or similarity of interpretability from the 30 raters. Only four items were considered problematic and needed a new translation. In terms of comparability of language, items 1, 5c, 8a, and 12d obtained a mean score of 3.13, 3.20,3.63, and 4.10, respectively; items $8 \mathrm{a}$ and $12 \mathrm{~d}$ obtained an interpretability mean score of 3.70 and 3.90 , respectively. After retranslating all the four problematic items, they were re-evaluated by 30 different raters, and each item obtained mean scores of three or less, allowing their use in the Spanish version of the questionnaire. After testing the 


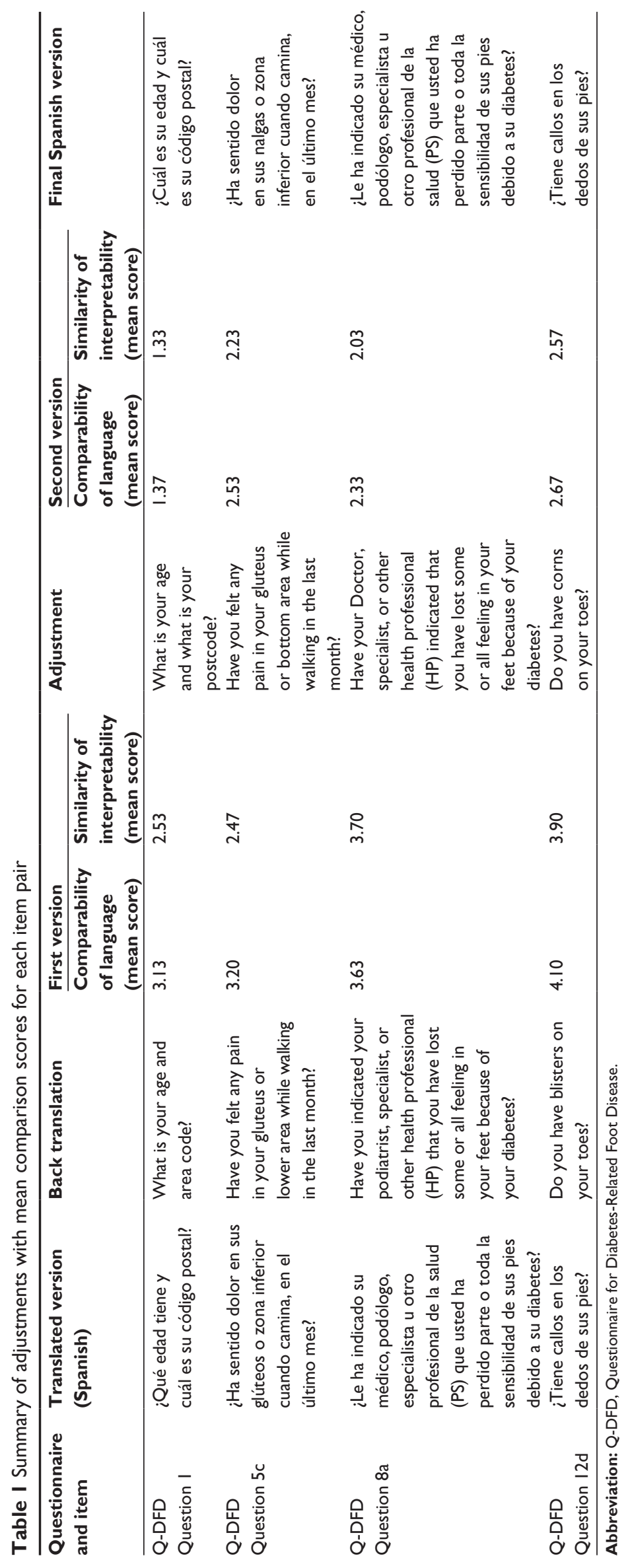


Q-DFD on 16 subjects, minimal adjustments were made, and the final Spanish version was created.

\section{Patient validation study \\ Criterion and construct validity}

A total of 92 patients were recruited and allocated into sample A $(\mathrm{n}=46)$ and sample B $(\mathrm{n}=46)$. In addition, sample B patients (B1) were divided into samples B2 and B3. Ten patients from sample A were excluded; five who could not be contacted after five repeated phone calls, two who refused to participate, two who did not attend the clinical assessment, and one who was critically ill at the time of the telephone call. In sample B, six patients were excluded: five due to unavailability and one who was recently diagnosed with Alzheimer's disease. Thus, in sample A and B, 36 (78\%) and 40 (87\%) patients, respectively, were ultimately included in the study. Participant characteristics are shown in Table 2.

For an overall diagnosis of DRFD (defined as presenting with at least one of DN, PVD, ulcer, deformity, or amputation), substantial level of agreement was obtained when we compared the Q-DFD with the clinical assessment (kappa 0.77 , sensitivity $80.4 \%$, specificity $91.5 \%$, LR+ 9.46, LR- 0.21), and an almost perfect level of agreement when compared with medical records (kappa 0.88, sensitivity $87 \%$, specificity $97 \%, L R+29.0, L R-0.13$ ). When we combined both, we obtained a kappa score of 0.82 , with a sensitivity of $83.5 \%$, specificity of $94.1 \%, \mathrm{LR}+$ of 14.1 , and LR- of 0.18 . This analysis was made without using deformity because it was not commonly recorded in medical records. Table 3 shows a summary of statistics regarding levels of agreement for samples A, B1, B2, and B3; with components of DRFD individually and combined.
The individual components of DRFD that showed the highest prevalence rates for community-based patients (sample A) were deformity and DN (75\% and 44.4\%), followed by PVD, ulcer, and amputation (16.7\%, 2.8\%, and $2.8 \%$ ). Deformity and DN were the most commonly reported components $(60 \%$ and $37.5 \%)$ for the clinic-based patients (sample B1), followed by ulcer, PVD, and amputation (27.5\%, $12.5 \%, 5 \%)$. A more detailed explanation of prevalence rates for sample B can be found in Table 4.

\section{Survey reliability}

A total of 41 patients completed the questionnaire on three different occasions. The first two interviews were made on the same day, and the third was completed 7 days later. Patient characteristics are shown in Table 2.

For an overall diagnosis of DRFD, intra-rater and interrater reliability obtained a substantial agreement, with a kappa score of 0.63 and 0.73 , respectively. Regarding interrater reliability, individual components of DRFD showed substantial to perfect agreement with DN (kappa $=0.76$ ), PVD (kappa $=0.72)$, ulcer $($ kappa $=1.0)$, and deformity $(\mathrm{kappa}=0.90)$. Furthermore, for intra-rater reliability, the individual components achieved moderate to perfect agreement with DN (kappa =0.69), PVD (kappa $=0.53)$, ulcer $($ kappa $=1.0)$, and deformity $($ kappa $=0.75)$. No analysis was made for amputation, as no individual reported this component.

\section{Discussion}

This study shows that the Spanish version of the Q-DFD is a valid and efficient diagnostic tool that allows the detection of DRFD in adults with DM. As hypothesized, the Spanish version of the Q-DFD showed good criterion and construct validity, and moderate to high intra- and inter-rater reliability.

Table 2 Participant characteristics used to determine validity and reliability of the survey

\begin{tabular}{|c|c|c|c|c|c|}
\hline & \multicolumn{5}{|l|}{ Sample } \\
\hline & $\bar{A}$ & B I & B2 & B3 & C \\
\hline Total participants & 36 & 40 & 18 & 22 & 41 \\
\hline \multicolumn{6}{|l|}{ Sex, n (\%) } \\
\hline Male & $13(36.1)$ & $21(52.5)$ & $9(50)$ & $12(54.5)$ & $16(39)$ \\
\hline Female & $23(63.9)$ & $19(47.5)$ & $9(50)$ & $10(45.5)$ & $25(6 \mathrm{I})$ \\
\hline \multicolumn{6}{|l|}{ Age, years } \\
\hline Mean \pm SD & $61.4 \pm 8.7$ & $66.6 \pm 9.6$ & $68.3 \pm 10.9$ & $65.1 \pm 7.8$ & $64.2 \pm 9.6$ \\
\hline Range & $48-85$ & $46-87$ & $48-87$ & $46-75$ & $50-89$ \\
\hline \multicolumn{6}{|c|}{ Diabetes duration, years } \\
\hline Mean \pm SD & $8.7 \pm 6.2$ & $18.4 \pm 10.1$ & $19.3 \pm 9$ & $17.6 \pm 10.8$ & $9.4 \pm 6.5$ \\
\hline Range & $\mid-23$ & $0.58-38$ & $6-33$ & $0.58-38$ & $0.5-23$ \\
\hline
\end{tabular}

Notes: Sample A was used to determine criterion validity, sample B was used to determine construct validity $(\mathrm{BI}=$ total clinic-based sample, B2 = clinic-based sample with foot complications, B3 = clinic-based sample without foot complications), sample $C$ was used to determine intra- and inter-rater reliability. Abbreviation: SD, standard deviation. 
Table 3 Summary of statistics used to determine criterion and construct validity

\begin{tabular}{|c|c|c|c|c|c|}
\hline & Kappa & Sensitivity \% & Specificity \% & LR+ & LR- \\
\hline \multicolumn{6}{|l|}{ combined (any diagnosis } \\
\hline \multicolumn{6}{|c|}{ Samples A and B combined (all components of DRFD) } \\
\hline DN & 0.728 & 0.839 & 0.889 & 7.559 & 0.181 \\
\hline PVD & 0.776 & 0.688 & 0.923 & 8.935 & 0.338 \\
\hline Ulcer & 0.856 & 0.846 & 0.969 & 27.290 & 0.159 \\
\hline Amputation & 1.000 & 1.000 & 1.000 & & \\
\hline \multicolumn{6}{|l|}{ Deformity ${ }^{\mathrm{a}}$} \\
\hline Sample A (any diagnosis of DRFD) & 0.774 & 0.804 & 0.915 & 9.459 & 0.214 \\
\hline \multicolumn{6}{|l|}{ Sample A (all components of DRFD) } \\
\hline $\mathrm{DN}$ & 0.550 & 0.750 & 0.800 & 3.750 & 0.313 \\
\hline PVD & 0.750 & 0.667 & 0.900 & 6.670 & 0.370 \\
\hline Ulcer & 0.654 & 0.500 & 0.971 & $17.24 \mid$ & 0.515 \\
\hline Amputation & 1.000 & 1.000 & 1.000 & & \\
\hline Deformity & 0.615 & 0.893 & 0.667 & 2.682 & 0.160 \\
\hline Sample BI (any diagnosis of DRFD) & 0.880 & 0.870 & 0.970 & 29.000 & 0.134 \\
\hline \multicolumn{6}{|l|}{ Sample BI (all components of DRFD) } \\
\hline DN & 0.893 & 0.933 & 0.960 & 23.325 & 0.070 \\
\hline PVD & 0.805 & $0.7 \mid 4$ & 0.943 & 12.526 & 0.303 \\
\hline Ulcer & 0.875 & 0.909 & 0.966 & 26.735 & 0.094 \\
\hline Amputation & 1.000 & 1.000 & 1.000 & & \\
\hline \multicolumn{6}{|l|}{ Deformity ${ }^{b}$} \\
\hline \multicolumn{6}{|c|}{ Sample B2 (complications group, all components of DRFD) } \\
\hline $\mathrm{DN}$ & 0.824 & 0.933 & 0.750 & 3.732 & 0.089 \\
\hline PVD & 0.753 & 0.714 & 0.846 & 4.636 & 0.338 \\
\hline Ulcer & 0.886 & 0.909 & 0.875 & 7.272 & 0.104 \\
\hline Amputation & 1.000 & 1.000 & 1.000 & & \\
\hline \multicolumn{6}{|l|}{ Deformity ${ }^{b}$} \\
\hline \multicolumn{6}{|c|}{ Sample B3 (no complications group, all components of DRFD) } \\
\hline $\mathrm{DN}$ & No respondent reported & 0.040 & 1.000 & & 0.960 \\
\hline PVD & No respondent reported & 1.000 & 1.000 & & \\
\hline Ulcer & No respondent reported & & & & \\
\hline Amputation & No respondent reported & & & & \\
\hline Deformity ${ }^{b}$ & & & & & \\
\hline
\end{tabular}

Notes: Sample A shows correlation between questionnaire and clinical evaluation, while sample B shows correlation between questionnaire and medical records (BI = total clinic-based sample, B2 = clinic-based sample with foot complications, B3 = clinic-based sample without foot complications). Patients were classed as having "any diagnosis of DRFD" if they presented with either one of DN, PVD, ulcer, amputation, or deformity. 'Deformity for sample A and B combined could not be obtained because it was not commonly recorded in the medical records of patients in sample B; beformity was not recorded in the medical records of patients in sample B.

Abbreviations: DN, diabetic neuropathy; DRFD, diabetes-related foot disease; LR+, positive likelihood ratio; LR-, negative likelihood ratio; PVD, peripheral vascular disease.

With English being the lingua franca of science, ${ }^{14}$ the need to adapt instruments for their use in other languages is of primary importance. However, it is not enough to just translate them; a cross-cultural adaptation of the instrument should also be performed. ${ }^{11}$ This is one of the strengths of our study. We also addressed criterion, construct validity, and survey reliability, which allowed us to obtain a successful cross-cultural adaptation, making the Q-DFD suitable for use in Spanish-speaking populations.

The first aim of the present study was to translate the Q-DFD from English into Spanish by following the international guidelines criteria proposed by Sperber. ${ }^{11}$ After translation, four items were considered problematic and were reviewed and retranslated. However, as can be seen in Table 1, we found that even after doing this, items $8 \mathrm{a}$ and $12 \mathrm{~d}$ of the first and final Spanish versions were identical. This could be related to the use of synonyms in the first and second English versions.

The second aim of the present study was to validate the Q-DFD in a diabetic population from Guayaquil. Regarding validation, we used the same methodology employed by Bergin et al. ${ }^{10}$ Our study had larger samples than those seen 
Table 4 Prevalence of individual components of diabetes-related foot disease according to the Q-DFD

\begin{tabular}{llllll}
\hline & \multicolumn{3}{l}{ Prevalence (\%) } \\
\cline { 2 - 6 } & DN & PVD & Ulcer & Amputation & Deformity \\
\hline Sample A & 44.4 & 16.7 & 2.8 & 2.8 & 75.0 \\
Sample BI & 37.5 & 12.5 & 27.5 & 5.0 & 60.0 \\
Sample B2 & 77.8 & 27.8 & 55.6 & 11.1 & 55.6 \\
Sample B3 & 4.5 & 0.0 & 4.5 & 0.0 & 63.6 \\
\hline
\end{tabular}

Notes: Sample A was used to determine criterion validity, sample $B$ was used to determine construct validity $(\mathrm{BI}=$ total clinic-based sample, $\mathrm{B} 2$ = clinic-based sample with foot complications, B3 = clinic-based sample without foot complications).

Abbreviations: DN, diabetic neuropathy; PVD, peripheral vascular disease; Q-DFD, Questionnaire for Diabetes-Related Foot Disease.

in the original article: sample A (36 versus [vs] 21), sample B (40 vs 25 ), and sample C (41 vs 30 ). Demographic characteristics were similar in terms of age distribution, with most of our patients in their 60s; but gender distribution differed, and mean diabetes duration was shorter.

In order to determine validity of the questionnaire, we tested the Q-DFD against the gold standard. In most of the components evaluated, our results were consistent with the findings from Bergin et $\mathrm{al},{ }^{10}$ which suggests the reproducibility of the original research and the validity of the Q-DFD to determine DRFD. Individually, items that showed the poorest levels of agreement were DN in sample A (kappa $=0.55$ ), and PVD in sample B (kappa $=0.81$ ); while amputation obtained perfect scores $(k a p p a=1.0)$ in both samples. This might be explained by the self-report of symptoms. DN and PVD are subjective components; in contrast to amputation. In the Q-DFD, the subjectivity of DN and PVD is taken into consideration by using questions to confirm that these symptoms are related to DRFD. For instance, questions $3 \mathrm{a}-\mathrm{e}$ are to determine whether patients have self-reported DN by asking for burning, tingling, numbness, tightness, and 'pins and needles' sensation. This self-reported DN is then confirmed by question 4 , where the patient should answer that the symptom(s) do not go away. Thus, question 4 assures us that symptoms answered in questions $3 \mathrm{a}-\mathrm{e}$ are persistent; a feature of DN. ${ }^{15}$

The Q-DFD showed high sensitivity and specificity, proving to be a diagnostic tool with good ability to predict the presence and absence of DRFD. In addition, the Q-DFD presented a high LR+ and a low LR-. This indicates that the Q-DFD performs well in excluding, as well as correctly detecting, DRFD. With regards to the prevalence of individual components of DRFD measured with the Q-DFD, our study shows that deformity is the most common finding in both samples (sample $A=75 \%$, sample $B=60 \%$ ); followed by DN (sample A $=44.4 \%$, sample $B=37.5 \%$ ). These data were similar to the original research by Bergin et al, ${ }^{10}$ but somewhat differ from other studies conducted in Latin America. For instance, Ibarra et a ${ }^{16}$ performed a study in Chile with 240 patients and determined a DN prevalence of $69 \%$; another study conducted in Mexico by Camacho López et a ${ }^{17}$ enrolled 207 patients and found a DN prevalence of 54.5\%. In addition, Tres et $\mathrm{al}^{18}$ assessed DN in 340 patients from Passo Fundo, a city in Southern Brazil, and obtained a prevalence of $22.1 \%$. We speculate that the reason for these results is that all the studies used different criteria to determine DN, highlighting the lack of universal guidelines.

When analyses of intra- and inter-rater reliability were performed, we obtained substantial levels of agreement for an overall diagnosis of DRFD. In addition, individual components of DRFD were also evaluated and showed moderate to perfect levels of agreement. We observed that ulcer and amputation scored higher than DN and PVD. As already mentioned, this could be explained by the objectivity of ulcer and amputation, in contrast to the subjectivity that DN and PVD present.

This study has some limitations that need to be considered. First, the recruitment of the clinic-based sample (sample B) was carried out in a single hospital. Thus, results cannot be extrapolated to the diabetic inpatient population from Guayaquil. Second, our participant selection was not random, which has led to a sample bias. Last, the assessment of DN was made using the pinprick test and not the $10 \mathrm{~g}$ Semmes-Weinstein Monofilament because it was not available in our city during clinical evaluation.

\section{Conclusion}

The results of the present study indicate that the translation of the Q-DFD is linguistically accurate and acceptable for use in Spanish-speaking populations. In addition, it showed good psychometric properties such as validity, reliability, and reproducibility. Although this questionnaire does not replace clinical examination, which is the gold standard of diagnosis, it has certainly been shown to be a simple and cost-effective method for the early detection of DRFD in populations that do not have access to health services.

\section{Acknowledgment}

The authors gratefully acknowledge Shan Bergin, $\mathrm{PhD}$, first author of the original Q-DFD in Australia, for her valuable help and guidance throughout this study. They also wish to thank Rodolfo Farfán, MD; Edmundo Estevez, MD; Tania Alvarado, MD; and José Barriga, MD for their cooperation in conducting this project. Furthermore, they thank the 
following medical students who conducted the interviews: Andrea Flores, Mayra Reyes, Daniela Salas, Cecilia Massuh, Dayanna Cabrera, Julia Larrea, José González, Mariuxi Coronel, María José Barrera, Buiyi Wong, Kiara Delgado, Erika Ramirez, Andrea Galecio, and Dajanne Cevallos.

The Spanish version of the Q-DFD can be obtained free of charge from the first author: Wilson Castillo-Tandazo: wcastillo@uees.edu.ec. The original English version of the Q-DFD may be obtained by contacting Shan M Bergin: shan. bergin@monashhealth.org.

\section{Disclosure}

All authors approved the final version of the manuscript and report no conflicts of interest in this work.

\section{References}

1. Shaw JE, Sicree RA, Zimmet PZ. Global estimates of the prevalence of diabetes for 2010 and 2030. Diabetes Res Clin Pract. 2010;87(1): 4-14.

2. World Health Organization. Diabetes programme. Country and regional data on diabetes. WHO region of the Americas. Prevalence of diabetes in the WHO Region of the Americas [webpage on the internet]. Available from: http://www.who.int/diabetes/facts/world_figures/en/index3.html. Accessed April 8, 2013.

3. INEC 2009. Statistical yearbook of Ecuador: vital statistics. Quito, Ecuador [01/5/13]; Available from: http://www.inec.gov.ec/web/guest/ inicio.

4. Khanolkar MP, Bain SC, Stephens JW. The diabetic foot. QJM. 2008;101(9):685-695.

5. Singh N, Armstrong DG, Lipsky BA. Preventing foot ulcers in patients with diabetes. JAMA. 2005;293(2):217-228.

6. Johannesson A, Larsson GU, Ramstrand N, Turkiewicz A, Wiréhn AB, Atroshi I. Incidence of lower-limb amputation in the diabetic and nondiabetic general population: a 10-year population-based cohort study of initial unilateral and contralateral amputations and reamputations. Diabetes Care. 2009;32(2):275-280.
7. Papazafiropoulou A, Tentolouris N, Soldatos RP, et al. Mortality in diabetic and nondiabetic patients after amputations performed from 1996 to 2005 in a tertiary hospital population: a 3-year follow-up study. J Diabetes Complications. 2009;23(1):7-11.

8. Laclé A, Valero-Juan LF. Diabetes-related lower-extremity amputation incidence and risk factors: a prospective seven-year study in Costa Rica. Rev Panam Salud Publica. 2012;32(3):192-198.

9. Cueva Recalde JF. [Validation of a new diabetic foot risk score in Quito (Equador).] Av Diabetol. 2009;25(6):486-490. Spanish [with English abstract].

10. Bergin SM, Brand CA, Colman PG, Campbell DA. A questionnaire for determining prevalence of diabetes related foot disease (Q-DFD): construction and validation. $J$ Foot Ankle Res. 2009;2:34.

11. Sperber AD. Translation and validation of study instruments for crosscultural research. Gastroenterology. 2004;126(1 Suppl 1):S124-S128.

12. Frykberg RG, Zgonis T, Armstrong DG, et al. Diabetic foot disorders. A clinical practice guideline (2006 revision). J Foot Ankle Surg. 2006;45(Suppl 5):S1-S66.

13. Landis JR, Koch GG. The measurement of observer agreement for categorical data. Biometrics. 1977;33(1):159-174.

14. Meneghini R, Packer AL. Is there science beyond English? Initiatives to increase the quality and visibility of non-English publications might help to break down language barriers in scientific communication. EMBO Rep. 2007;8(2):112-116.

15. Archer AG, Watkins PJ, Thomas PK, Sharma AK, Payan J. The natural history of acute painful neuropathy in diabetes mellitus. J Neurol Neurosurg Psychiatry. 1983;46(6):491-499.

16. Ibarra CT, Rocha Jde J, Hernández RO, Nieves RE, Leyva RJ. [Prevalence of peripheral neuropathy amongh primary care type 2 diabetic patients.] Rev Med Chil. 2012;140:1126-1131. Spanish [with English abstract].

17. Camacho López J. Prevalencia de neuropatía periférica en pacientes con diabetes mellitus tipo 2, atendidos en la Clínica Hospital del ISSSTE en Mazatlán, Sinaloa. [Prevalence of peripheral neuropathy among type 2 diabetic patients attending the hospital clinic ISSSTE in Mazatlán, Sinaloa.] Rev Esp Med Quir. 2011;16(2):71-74. [with English abstract].

18. Tres GS, Lisbôa HR, Syllos R, Canani LH, Gross JL. Prevalence and characteristics of diabetic polyneuropathy in Passo Fundo, South of Brazil. Arq Bras Endocrinol Metabol. 2007;51:987-992.
Vascular Health and Risk Management

\section{Publish your work in this journal}

Vascular Health and Risk Management is an international, peerreviewed journal of therapeutics and risk management, focusing on concise rapid reporting of clinical studies on the processes involved in the maintenance of vascular health; the monitoring, prevention and treatment of vascular disease and its sequelae; and the involvement of

\section{Dovepress}

metabolic disorders, particularly diabetes. This journal is indexed on PubMed Central and MedLine. The manuscript management system is completely online and includes a very quick and fair peer-review system, which is all easy to use. Visit http://www.dovepress.com/ testimonials.php to read real quotes from published authors. 